\title{
Functional foods: a simple scheme for establishing the scientific validity for all claims
}

\author{
Margaret Ashwell OBE \\ Ashwell Associates, Ashwell Street, Ashwell, Hertfordshire, SG7 5PZ, UK
}

'Functional food' has become a buzzword in the Food Industry and many conferences and new journals are now including these words in their titles. Recent estimates suggest that the market for functional foods is approximately $£ 830$ million in Europe, predicted to jump to £1.6 billion by 2010, and that the global value of this sector could potentially be equivalent to $5 \%$ of the world food market $^{1}$.

My personal, maybe somewhat sceptical, view is that there is nothing terribly new about most of the science behind functional foods. In fact if nutritionists had employed a public relations company, probably at great expense, to promote the importance of the positive aspects of nutrition science, they couldn't have come up with a better idea! So why not let us use this as a great opportunity to emphasise the importance of scientific substantiation of the claims being made for these foods, to help us emphasise the importance of the scientific approach to nutrition? After all, the vast majority of the general public will only learn about nutrition from the claims made on food packets and in media advertisements. If we can promote a process for scientific support for health-related claims for foods and food components then we will be making a great contribution to consumer education.

Roberfroid $^{2}$ sees the science behind functional foods as a natural, and essential, evolution from traditional nutrition science. During the twentieth century, nutritionists discovered the essential nutrients and they established nutrient standards, dietary guidelines and food guides, mainly, if not exclusively, with the aim of preventing deficiencies and of supporting body growth, maintenance and development. More recently they have also made recommendations aiming to avoid excessive consumption of some of these nutrients after recognising their potential role in the aetiology of miscellaneous (mostly chronic) diseases.

At the turn of the twenty-first century, in the society of abundance which characterises most of the occidental/ industrialised world, we face new challenges from uncontrollable increase in the costs of health care, increase in life expectancy, improved scientific knowledge and development of new technologies to major changes in lifestyles. Roberfroid believes that nutrition has to adapt to these new challenges. As a consequence, nutrition as a science in the twenty-first century will, in addition to placing emphasis on balanced diet, develop the concept of optimum nutrition. On the road to optimum nutrition, which is an ambitious and long-term objective, functional food science is a new, interesting and stimulating concept that is aimed at improving dietary guidelines by integrating new knowledge on the interactions between food components and body functions and/ or pathological processes.

The concept of Foods for Specified Health Use (FOSHU) was established in Japan in 1991. These foods are included as one of the four categories of foods, described in the 'Nutrition Improvement Law', as 'Foods for special dietary use' (i.e. 'foods that are used to improve people's health and for which specific health effects are allowed to be displayed'). Upon satisfactory submission of comprehensive data documenting the scientific evidence in support of a proposed health claim, the Minister of Health and Welfare is able to approve a claim, and grant permission, to use a 'symbol' on labelling to indicate to the consumer that the health claim has government approval. Foods identified as FOSHU are required to provide evidence that the final food product is expected to exert a health or physiological effect; data on the effects of isolated individual components are not sufficient. FOSHU products should be in the form of ordinary foods (i.e. not as pills or capsules) and are assumed to be consumed as part of an ordinary diet (i.e. not as very occasional items linked to specific symptoms). Most FOSHU products currently approved contain either oligosaccharides or lactic acid bacteria for promoting intestinal health.

In the United States of America, 'reduction of disease risk' claims have been allowed since 1993 on certain foods. These contain components for which the Food and Drug Administration (FDA) has accepted there is objective evidence for a correlation between nutrients or foods in 
the diet and certain diseases on the basis of 'the totality of publicly available scientific evidence, and where there is substantial agreement amongst qualified experts that the claims were supported by the evidence'. By the end of 1999 there were 12 FDA-approved correlations between foods, or components, and diseases. The FDA also allows claims to be based on 'authoritative statements' of a Federal Scientific Body, such as the National Institutes of Health and Center for Disease Control, as well as from the National Academy of Sciences - as allowed by the FDA Modernization Act of 1997, and is considering a process whereby a company can apply to the FDA in respect to a particular nutrition and health link they wish to claim. The company would fund studies to develop evidence for their proposed claim and the FDA would 'direct' this research to monitor its quality. The FDA has published a document as Guidance for Industry that contains scientific principles for those designing studies to support health claims petitions ${ }^{3}$. This is a very useful document that covers identifying data for review, performing reliable measurements, evaluating individual studies, evaluating the totality of the evidence and assessing significant scientific agreement.

In the European Union, there is no harmonised legislation on health claims, which means that they are dealt with at a national level. Not surprisingly, there have already been several calls for better regulation of functional and health claims for foods and supplements ${ }^{4}$. It is therefore up to public health nutritionists to ensure that a proper scientific basis for these claims is established as soon as possible.

So, what are functional foods? The recent Consensus Document on scientific concepts of functional foods in Europe $^{5}$ was an important development in this area. Appendix A shows the key definitions from the Document.

However, the main thrust of the Consensus Document ${ }^{5}$ was to suggest a novel scheme whereby claims for functional foods should be linked to solid scientific evidence, based on markers.

In 1999, Codex Alimentarius ${ }^{6}$ proposed Draft recommendations for the use of health claims and has identified two types of claim. 'Enhanced Function Claims' concern specific beneficial effects of the consumption of foods and their constituents on physiological (or psychological) functions or biological activities but do not include nutrient function claims. Such claims relate to a positive contribution to health or to a condition linked to health to the improvement of a function or to modifying or preserving health. 'Reduction of Disease Risk Claims' are 'for reduction of disease risk related to the consumption of a food or food constituent in the context of the daily diet that might help reduce the risk of a specific disease or condition'. Although amendments to these definitions are now the subject of consultation, the distinction between the two types of claim is still preserved ${ }^{7}$.
The Codex proposals bear a pleasing congruence with the Type A (Enhanced Function Claims) and Type B (Reduced Risk Of Disease Claims) suggested in the Consensus Document ${ }^{5}$. This proposed that claims should be based on evidence related to markers which are linked to clearly defined and measurable outcomes and are significantly and consistently modulated in rigorously controlled studies by the particular food component.

Enhanced Function Claims should be accompanied by evidence based on valid, reproducible, sensitive and specific markers relating to the target function or biological response, such as changes in body fluid levels of a metabolite, protein or enzyme (e.g. the reduction in levels of plasma homocysteine as a response to dietary folate, or the increased levels of brain serotonin as a response to dietary tryptophan).

Reduction Of Disease Risk Claims, however, would only be justified if the evidence is based on valid, reproducible, sensitive and specific markers relating to an appropriate intermediate endpoint of an improved state of health and well-being and/or reduction of risk of disease, such as the measurement of a biological process which relates directly to the endpoint (e.g. the extent of narrowing of the carotid artery as evidence of cardiovascular disease; or functional imaging of the brain by magnetic resonance imaging as an intermediate endpoint marker for the amelioration of depression).

This suggestion that the type of claim made should relate to the type of marker was indeed a novel approach to evaluating the scientific support for health-related claims for foods and food components. The FDA document ${ }^{3}$ gives guidance on which acceptable biomarkers can be used to support health claims but it does not go further than this.

The new Codex proposals, if accepted, will ultimately be incorporated into their existing Guidelines ${ }^{6}$ where four different types of nutrition claim have already been defined. One of these is the nutrient content claim that refers to the level of a nutrient contained in a food, such as 'Source of calcium' or 'Rich in folic acid'. At present, there is legislation to ensure that nutrient content claims for vitamins and minerals are only made if the food contains a significant amount of micronutrient in relation to its Recommended Daily Allowance ('labelling' RDA) ${ }^{8}$. There has never been a requirement to show that the nutrient in the food is actually biologically accessible to the body, and it has always been assumed that any additional nutrient which might be added as a fortificant behaves in the same way as the nutrient found naturally in the food.

To a small extent, the existing legislation for vitamins and minerals takes biological accessibility into account because RDAs are recommendations of intakes that are calculated from knowledge of population requirements and the average bioavailability of that nutrient in 'normal' 
foods. However, very little evidence exists for the biological accessibility of vitamins and minerals in fortified foods, let alone other potential components in functional foods such as flavonoids or phytoestrogens. If all claims are to have a scientific base, then it should not be enough just to state that a functional food contains a certain amount of a food component. The biological accessibility of that component within that functional food should be demonstrated. In the case of functional food components that are purported to act intracellularly, this will require the demonstration of absorption of the functional food component through the intestinal wall at least into the bloodstream. In the case of functional food components that act within the gut, this would require the demonstration of their presence at the site of action.

If the logic from the Consensus Document is extended to its extreme, then my personal view is that 'nutrient content' claims, particularly if applied to 'novel' functional food components, should be based not only on evidence for presence of the component in the food but also on scientific evidence using markers of exposure that indicate the biological accessibility of the active component, such as its delivery to the intestine or its absorption through the intestinal wall and into the relevant cells, as appropriate.

Several European countries have instigated self-regulating programmes for 'health claims' and the Joint Health Claims Initiative has now been launched in the UK. These Codes all encourage companies wanting to make innovative health claims to submit full documentation of the scientific evidence that forms the basis of the claim.

My proposal (as summarised in Appendix B) is an extension to the scheme in the Consensus Document ${ }^{5}$ and it offers a simple scientific framework for the preparation of 'support dossiers', as well as a broad framework for the regulation of all claims (nutrient claims and health claims). It is intended to be used in conjunction with the comprehensive FDA Guidance ${ }^{3}$ or similar Codes of Practice which cover the issues of evaluating the totality of the evidence and assessing significant scientific agreement.

Public health nutritionists should encourage and help such companies to think along these lines. Not only will this help the Food Industry to gain a good reputation for making truthful claims, it will also help to preserve a good reputation for the science of nutrition and the people who work within it. If the same people can also be play an important role in educating consumers or advising the people who educate consumers, then a consistent message will be promulgated. This must be a long-term objective for improving public health.

\section{References}

1 Potter D. Total integration. Functional Foods and Nutraceuticals 1999; 2(10): 30-2.

2 Roberfroid M. Defining functional foods. In: Gibson G, Williams C, eds. Functional Foods. Cambridge: Woodhead Publishing Ltd, 2000.

3 US Food and Drug Administration. Guidance for Industry Significant Scientific Agreement in the Review of Health Claims for Conventional Foods and Dietary Supplements. Washington, DC: US Department of Health and Human Services, 1999.

4 Katan M. Functional foods. Lancet 1999; 354(Sept 4): 794.

5 Diplock A, Aggett P, Ashwell M, et al. Scientific concepts of functional foods in Europe: Consensus Document. Br. J. Nutr. 1999; 81(4): S1-27.

6 Codex Alimentarius. Proposed Draft Recommendations for the Use of Health Claims. Alinorm 01/22, Appendix VIII. Vol. 99/ 22A Appendix VII. Geneva: World Health Organization, 1999.

7 Codex Alimentarius. Proposed Draft Guidelines for the Use of Health and Nutrition Claims. Alinorm 01/22, Appendix VIII. Vol. 99/22A Appendix VII. Geneva: World Health Organization, 2000.

8 Statutory Instruments. The Food Labelling Regulations 1996, Vol. 1499. London: HMSO, 1996.

\section{Appendix A: Definitions of functional foods (from Consensus Document ${ }^{5}$ )}

- No universally accepted definition for functional foods exists. In fact, because functional foods are more of a concept than a well-defined group of food products, a working definition rather than a firm definition is preferred for the purposes of this Consensus Document.

- A food can be regarded as 'functional' if it is satisfactorily demonstrated to affect beneficially one or more target functions in the body, beyond adequate nutritional effects in a way which is relevant to either an improved state of health and well-being and/or reduction of risk of disease. Functional foods must remain foods and they must demonstrate their effects in amounts which can normally be expected to be consumed in the diet. They are not pills or capsules, but part of a normal food pattern.

- A functional food can be a natural food, a food to which a component has been added, or a food from which a component has been removed by technological or biotechnological means. It can also be a food where the nature of one or more components has been modified, or a food in which the bioavailability of one or more components has been modified; or any combination of these possibilities. A functional food might be functional for all members of a population or for particular groups of the population which might be defined, for example, by age or by genetic constitution. 
Appendix B

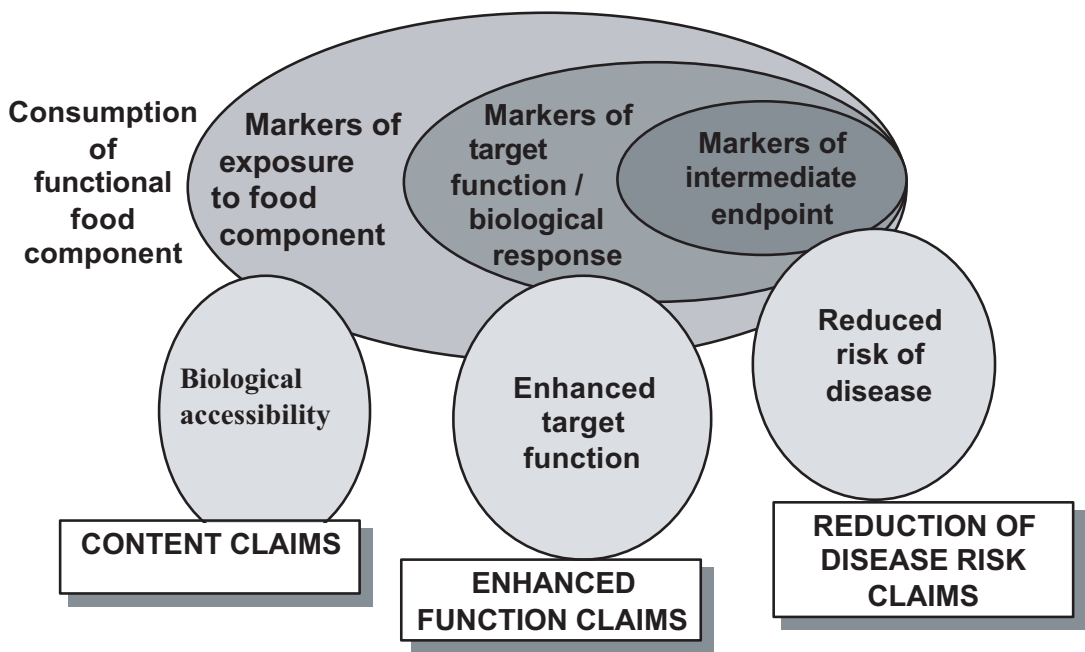

